\title{
Shot noise limited heterodyne detection of CARS signals
}

\author{
M. Jurna, J.P. Korterik, C. Otto*, and H.L. Offerhaus \\ Optical Techniques group, MESA ${ }^{+}$Institute for Nanotechnology, Faculty of Science and \\ Technology (TNW), University of Twente, the Netherlands \\ ${ }^{*}$ BioPhysical Engineering group, MESA ${ }^{+}$Institute for Nanotechnology, Faculty of Science \\ and Technology (TNW), University of Twente, the Netherlands
}

H.L.Offerhaus@TNW.Utwente.nl

\begin{abstract}
We demonstrate heterodyne detection of CARS signals using a cascaded phase-preserving chain to generate the CARS input wavelengths and a coherent local oscillator. The heterodyne amplification by the local oscillator reveals a window for shot noise limited detection before the signal-to-noise is limited by amplitude fluctuations. We demonstrate an improvement in sensitivity by more than 3 orders of magnitude for detection using a photodiode. This will enable CARS microscopy to reveal concentrations below the current mMolar range.
\end{abstract}

(C) 2007 Optical Society of America

OCIS codes: (190.4410) Nonlinear optics, parametric processes; (300.6230) Spectroscopy, coherent anti-Stokes Raman scattering; (300.6290) Spectroscopy, four-wave mixing; (300.6310) Spectroscopy, heterodyne.

\section{References and links}

1. J. X. Cheng, A. Volkmer, and X. S. Xie, "Theoretical and experimental characterization of coherent anti-Stokes Raman scattering microscopy,” J. Opt. Soc. Am. B 19,, 1363-1375 (2002).

2. A. Voroshilov, C. Otto, and J. Greve, "Secondary Structure of Bovine Albumin as Studied by PolarizationSensitive Multiplex CARS Spectroscopy," Appl. Spectrosc. 50,, 78-85 (1996).

3. G. Petrov and V. Yakovlev, "Enhancing red-shifted white-light continuum generation in optical fibers for applications in nonlinear Raman microscopy," Opt. Express 13, 1299-1306 (2005).

4. H. Kano and H. Hamaguchi, "In-vivo multi-nonlinear optical imaging of a living cell using a supercontinuum light source generated from a photonic crystal fiber," Opt. Express 14,, 2798-2804 (2006).

5. X. Nan, E. Potma, X.S. Xie, "Nonperturbabtive chemical imaging of organelle transport in living cells with CARS microscopy," Biophysical J. 91, 728-735 (2006).

6. B. Rakic, S.M. Sagan, M. Noestheden, S. Belanger, X. Nan, C.L. Evans, X.S. Xie and J.P. Pezacki, "Peroxisome peroliferator-activated receptor $\alpha$ antagonism inhibits hepatitis $C$ virus replication," Chem. Biol. 13, 23-30 (2006).

7. E. O. Potma, C. L. Evans, and X. S. Xie, "Heterodyne coherent anti-Stokes Raman scattering (CARS) imaging," Opt. Lett. 31, 241-243 (2006).

8. R.W. Boyd, "Nonlinear Optics, second edition", Academic press, ISBN-13:978-0-12-121682-5, ISBN-10:0-12121682-9

9. M. Jurna, J.P. Korterik, H.L. Offerhaus and C. Otto, "Noncritical phase-matched lithium triborate optical parametric oscillator for high resolution coherent anti-Stokes Raman scattering spectroscopy and microscopy," Appl. Phys. Lett. 89, 251116 (2006).

10. T. Tukker, "A high repetition rate picosecond infrared light source for a sum frequency vibration spectrometer," Thesis University of Twente, The Netherlands, ISBN 90-365 11860 (1998).

11. C.L. Evans, E.O. Potma, M. Puoris'haag, D. Cote, C.P. Lin, and X. S. Xie, "Chemical imaging of tissue in vivo with video-rate coherent anti-Stokes Raman scattering microscopy," Proc. Natl. Acad. Sci. USA 102, 1680716812 (2005).

12. J.X. Cheng, Y.K. Jia, G.F. Zheng, and X.S. Xie, "Laser-scanning coherent anti-stokes Raman scattering microscopy,” Biophys. J. 83, 502-509 (2002). 
13. E.R. Andresen, S.R. Keiding, E.O. Potma, "Picosecond anti-Stokes generation in a photonic-crystal fiber for interferometric CARS microscopy," Opt. Express 14, 7246-7251 (2006).

14. B. von Vacano, T. Buckup and M. Motzkus, "Highly sensitive single-beam heterodyne coherent anti-Stokes Raman scattering," Opt. Lett. 31, 2495-2498 (2006).

15. E.M.H.P. van Dijk, "Single Nanoparticles, ultrafast and unltrasensitive detection," Thesis Universiteit Twente, The Netherlands ISBN 90-365-2136-X (2005).

\section{Introduction}

By probing the internal vibrational structure of molecules, the CARS process enables label-free identification and chemically selective microscopy. The resonant enhancement of the CARS process makes it faster than Raman and compared to IR spectroscopy the short wavelengths involved, ensure a higher spatial resolution. CARS spectroscopy and microscopy have therefore attracted considerable attention [1, 2, 3, 4]. Implementation has been limited due to the complexity of the required systems and the low yield of the highly nonlinear interaction. The low CARS yield limits it to detection of substances with concentrations in the mMolar regime. Fluorescence spectroscopy of labeled species offers higher signal levels for situations where the autofluorescence is low and introduction of (large, disruptive and often toxic) labels can be tolerated. In biological and food-related systems, autofluorescence is generally high and labels are preferably avoided but mMolar concentrations are unrealistically high for many of the interesting substances $[5,6]$.

In this paper we demonstrate that CARS spectroscopy is possible at the shot noise limit using a compact setup that exploits the phase coherence of the CARS process for heterodyne detection [7]. The setup includes an OPO in which a signal and idler wavelength are created. The phase of the signal and idler individually are undetermined. The idler is used for the generation of the CARS whereas the signal is used as the local oscillator. Although these wavelengths bear no direct phase relation to each other, we demonstrate that the CARS output wavelength is phase-locked to the signal wavelength creating an appropriate scheme for heterodyne detection. Analysis of the noise properties of the local oscillator reveal a window that allows for shot noise limited detection of CARS signals. Attenuation measurements demonstrate the detection of CARS signal levels well below the detector dark (noise) level and the current detection limits. The setup is inherently jitter-free and makes use of every single wavelength that is generated.

Figure 1 depicts the phase preserving nonlinear chain. We start with a passively modelocked Nd:YAG laser (Vanguard by Spectra Physics or a Paladin by Coherent) generating $~ 15 p s$ pulses at a repetition rate close to $80 \mathrm{Mhz}$. The output at $1064 \mathrm{~nm}$ is partially doubled to pulses of $12 \mathrm{ps}$ at $532 \mathrm{~nm}$. This doubling is phase coherent [8] where the phase of the $532 \mathrm{~nm}$ is given by the addition of the phases of the two photons that make up the $532 \mathrm{~nm}$. The $532 \mathrm{~nm}$ synchronously pumps an Optical Parametric Oscillator (OPO) $[9,10]$ generating pulses of $\sim 6.5 p s$ at the signal and idler wavelength. The phase of the signal and idler separately are free but the addition of their phases is locked to the phase of the pump. The freedom for the phase of the signal ensures that the resonating signal has no phase restrictions and projects the carrier envelope phase slips and any other phase errors onto the non-resonating idler, ensuring smooth operation. The idler wavelength is combined with the $1064 \mathrm{~nm}$ to generate a CARS signal at $\omega_{\text {CARS }}=$ $2 \omega_{\text {pump }}-\omega_{\text {Stokes }}$, where the $1064 \mathrm{~nm}$ acts as the pump and the idler as the Stokes wavelength. The phase of the signal is given by $\left(2 \times\right.$ phase $_{1064}-$ phase $\left._{\text {idler }}\right)$. The CARS (or anti-Stokes) wavelength is equal to the signal wavelength from the OPO. Moreover, the phase of the resonant CARS signal is determined as $\left(2 \times\right.$ phase $_{1064}-$ phase $_{i d l e r}+$ phase $\left._{\chi^{(3)}}\right)$, where the last term is a constant for a fixed wavelength. The phase of the signal is therefore locked to the phase of the CARS signal except for path-length variations between the point of creation and the point where they interfere on the detector. The phase preservation means that the signal wavelength can interfere with the CARS signal in a predictable way and can thus be used for interferometric

\#83423 - \$15.00 USD

(C) 2007 OSA 


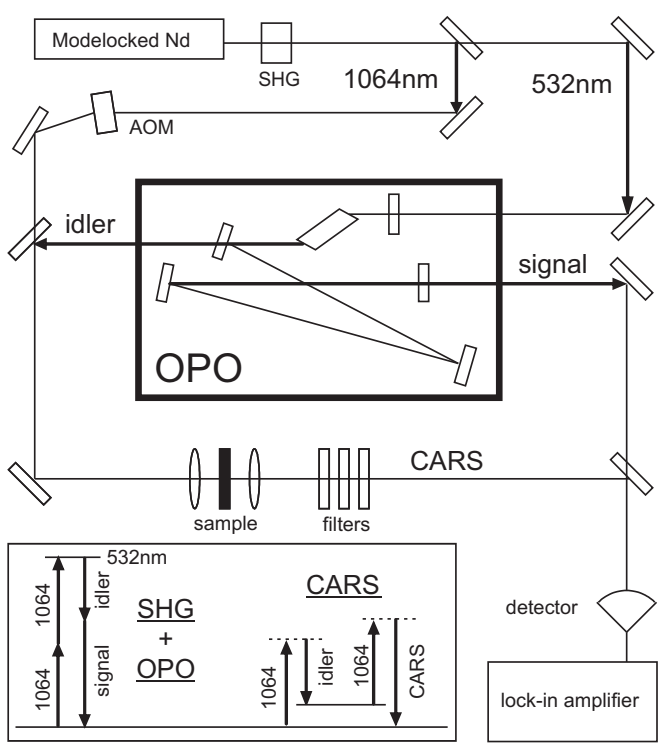

Fig. 1. Optical chain for the phase-preserved generation of wavelengths for the CARS process. The inset shows the energy diagram for both paths to the CARS wavelength

amplification.

\section{Heterodyne detection}

The interference of the Local Oscillator (LO, the OPO signal wavelength) with the CARS signal yields a total signal intensity on the detector of

$$
I_{\text {detector }}=L O+C A R S+\underbrace{2 \cdot \sqrt{L O \cdot C A R S}}_{H C A R S}
$$

where LO and CARS signify intensities. HCARS refers to the Heterodyne CARS power. This interference term scales with the root of the LO power and the interferometric gain can be defined as $H C A R S / C A R S=2 \sqrt{L O / C A R S}$. The noise in the optical signal is determined by the shot noise in the CARS signal itself and the shot noise introduced by the local oscillator. The introduced shot noise is the dominant term of the two (as $L O \gg C A R S$ ) and scales with the root of LO, just like the interference term and the interferometric gain. The interferometric amplification can thus be used to lift the signal above the detector noise without degrading the original signal-to-noise ratio as long as the local oscillator shot noise is the dominant term. To avoid $1 / f$ noise, we bring the detection to a spectrally less noisy region, shifting the CARS wavelength $40 \mathrm{kHz}$ with respect to the OPO signal wavelength.

The detector can be either a Photo-Multiplier Tube (PMT), Photo-Diode (PD) or Avalanche Photo Diode (APD). APD's are generally used in photon counting mode, generating a pulse for each detected photon. The PMT and PD are connected to a transimpedance amplifier terminated at zero Ohm by an OPerational AMPlifier (OPAMP), as depicted in the inset in figure 2. The PD can be considered as a current source with a current proportional to the detected intensity (typically $0.6 \mathrm{~A} / \mathrm{W}$ ). The dominant noise sources in the detector are the Johnson noise in the resistor ( $\sqrt{4 k T R \cdot B W}$ where $B W$ represents the detection bandwidth in $H z)$, the amplifier current noise (dark current independent of $R$ ) and the shot noise of the number of electrons generated by the detected signal. The detected voltage scales linear with the transimpedance 


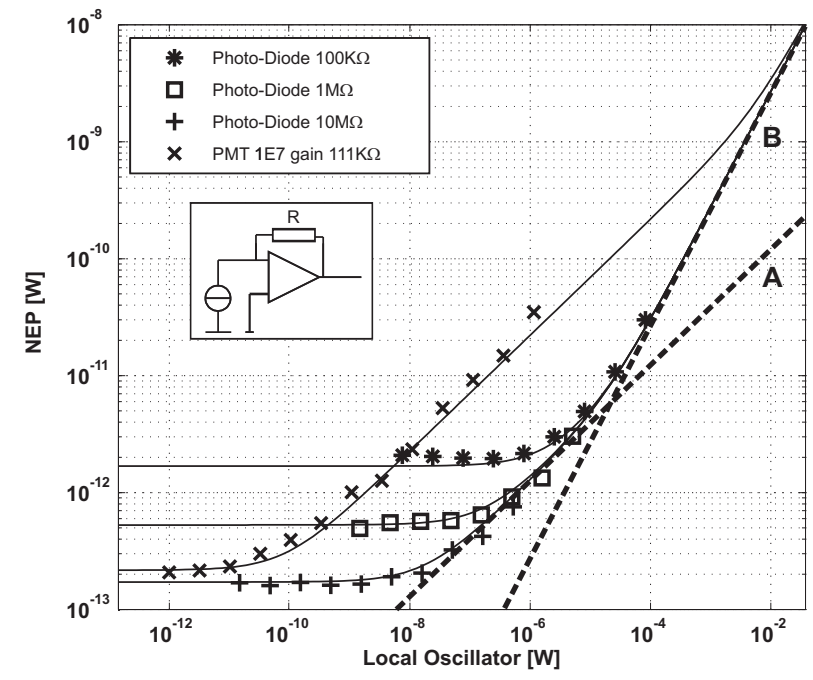

Fig. 2. Noise as a function of local oscillator power. The noise is expressed in Noise Equivalent Power (NEP), Root Mean Square (rms) in $1.58 \mathrm{~Hz}$ bandwidth so that all lines can be compared directly. The dashed lines A and B have a slope of $1 / 2,1$ respectively. Continuous lines are a simulation, symbols represent measured data. Inset shows the detector electronics.

resistor $(R)$. By increasing the resistor to the point where it dominates the other noise sources, the signal-to-noise of the amplifier scales with $\sqrt{R}$ and for sufficiently large output, the noise is dominated by the shot noise of the detected signal. The maximum $R$ is limited by the required bandwidth of the amplifier which scales as $1 / R$. A typical configuration yields a $1 M H z$ bandwidth for $1 M \Omega$ transimpedance. The PMT is similar to the PD except that the dark current is lower when compared to a resistor of similar gain. At a gain of $10 M$ a PMT typically has some equivalent 100photons $\cdot B W$ dark count noise (10k/s dark counts). APDs have the lowest dark count rate $(\simeq 10$ photons $\cdot B W)$.

When the amount of local oscillator power on the detector is increased, the noise (signal without any CARS signal on the detector) first shows the dark count noise level (flat) followed by a section showing the shot noise of the local oscillator. This shows up as a slope of $1 / 2$ in a log-log plot of the noise versus the LO power (parallel to line A in fig 2). At some point the noise becomes dominated by the amplitude fluctuations of the local oscillator. The amplitude fluctuation are correlated to the local oscillator power and therefore scale with a slope of 1 (parallel to line B). Since this section of the noise scales faster than the heterodyne signal (scales parallel to line A), the signal-to-noise deteriorates. The section in which the noise follows line A is the window in which CARS signals can be detected limited only by the shot noise.

The gain dynamics in the OPO determine the amplitude fluctuations, in particular the saturation level. If the OPO is not strongly saturated the amplitude fluctuations can show up at (local oscillator) levels where the noise is not yet dominated by the shot noise, effectively closing the window for shot noise limited detection.

The level of the detected shot noise compared to the "real" optical shot noise scales inversely with the root of the Quantum Efficiency $(\mathrm{QE})$, shifting that portion of the line upward. The position of the amplitude fluctuations is unaffected as it scales directly with the number of photons. This seems to improve the detection window by allowing higher levels of local oscillator but in reality deteriorates the detection sensitivity (see figure 4). PMTs tend to have a low QE ( $10 \%$ to $0.1 \%$ ) for wavelengths towards the Near Infra Red (NIR) that are traditionally favored 


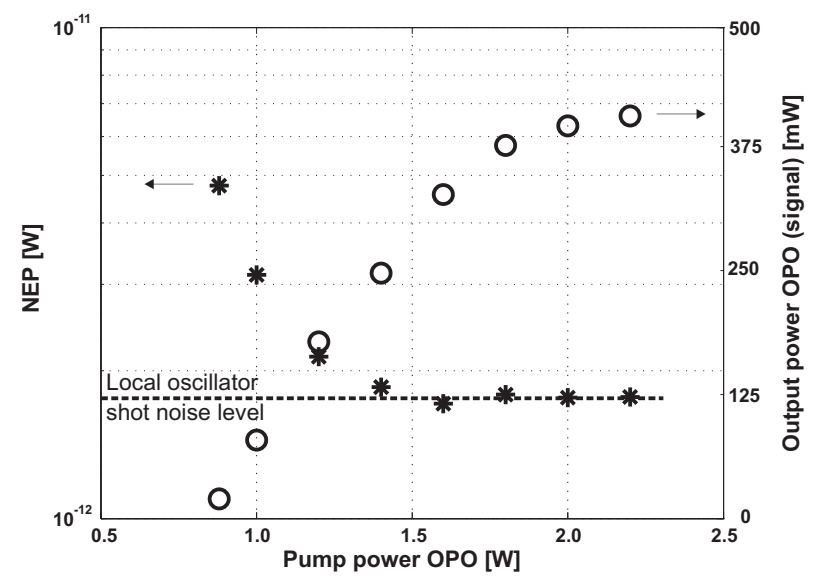

Fig. 3. Noise (NEP, $\mathrm{R}=1 \mathrm{M} \Omega, \mathrm{BW}=1.58 \mathrm{~Hz}$ ) for $2 \mu \mathrm{W}$ of the signal and the total signal output versus OPO pump power (532nm inside the crystal)

for biological CARS detection [11, 12]. PDs and APDs have a much better QE (typically up to $85 \%$ ). For the detection of very low signals therefore APDs are best. For practical applications PDs are favored because they are large, cheap and robust. APDs are expensive and fragile and suffer from a reduced dynamic range. As long as a window exists for shot noise limited detection, the detector choice is irrelevant.

\section{Results and Discussion}

We have tuned the OPO idler to $1578 \mathrm{~nm}$ so that the difference frequency between the $1064 \mathrm{~nm}$ and $1578 \mathrm{~nm}$ matches the $C-H$ stretch vibration at $3060 \mathrm{~cm}^{-1}$ in toluene. The OPO signal (and CARS signal) is at $802.7 \mathrm{~nm}$. The toluene sample is prepared between two cover glasses and is approximately $15 \mu \mathrm{m}$ thick. Tens of mWatts of $1064 \mathrm{~nm}$ and $1572 \mathrm{~nm}$ are focused using a $0.60 \mathrm{NA}$ air objective. The CARS signal is collected with an 0.65NA objective. Filters then remove the $1578 \mathrm{~nm}$ and $1064 \mathrm{~nm}$.

To shift the CARS frequency we use an AOM that is placed in the $1064 \mathrm{~nm}$, rather than the signal beam, because this wavelength does not change as the OPO is tuned for a particular vibrational frequency. The AOM is driven at $80.00 \mathrm{Mhz}$, effectively shifting the $1064 \mathrm{~nm}$ pulses, at a repetition rate of $80.02 \mathrm{Mhz}$ (depending on the room temperature), by $20 \mathrm{kHz}$. This shift is translated to a shift of $40 \mathrm{kHz}$ at the CARS wavelength due to the involvement of two photons at $1064 \mathrm{~nm}$ in the creation process. The CARS wavelength is combined on a detector with the OPO signal wavelength and the detected intensity is fed to a lock-in amplifier set to detect at $40 \mathrm{kHz}$. We integrate for $100 \mathrm{~ms}$ using a second order cut-off filter $(B W=1.58 \mathrm{~Hz})$.

Figure 2 shows the detected noise levels for a photodiode with three different transimpedances and a PMT as a function of the local oscillator power. The noise levels are back calculated to noise equivalent input power (at $803 \mathrm{~nm}, 1.58 \mathrm{~Hz}$ bandwidth) for comparison. The shot noise line for the PMT lies substantially above that for the PDs due to the low quantum efficiency $(0.3 \%)$. The PMT shows a large window for shot noise limited detection. The PD shows a window only when terminated by $1 M \Omega$ or $10 M \Omega$. At lower termination the required local oscillator level introduces noise dominated by amplitude fluctuations. Above $10 M \Omega$ the opamp is no longer able to follow the $40 \mathrm{KHz}$ oscillations.

The dependence of the amplitude fluctuations level on the saturation of the OPO is demonstrated in figure 3 where we plot both the OPO (signal) output power and the detected noise at 


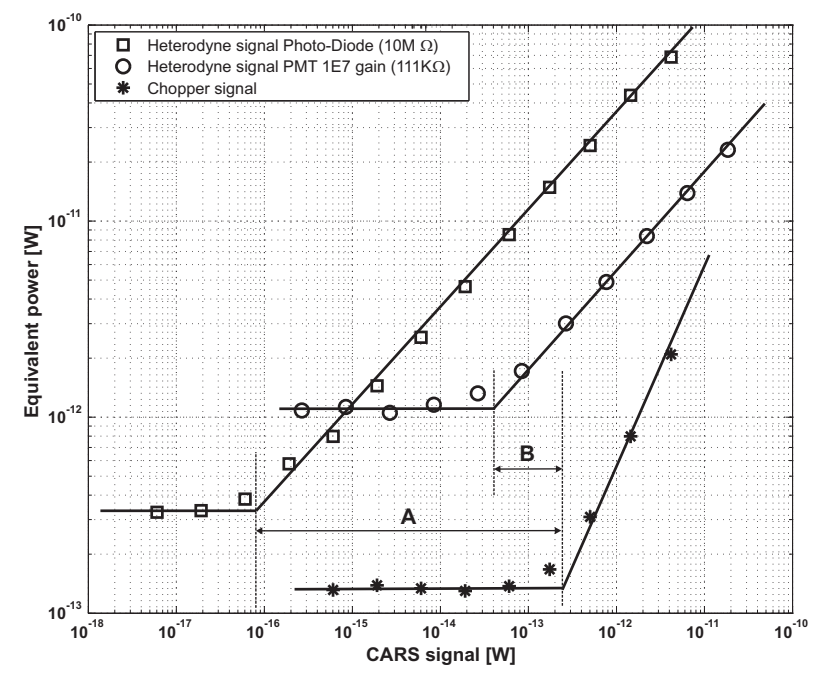

Fig. 4. Heterodyne signal and direct detected signal (rms in $1.58 \mathrm{~Hz} \mathrm{BW}$ ) versus CARS power. (A) represents the improvement factor (3500) in detection sensitivity between the heterodyne detection and direct (chopper) detection signal for a photodiode. (B) represents the improvement factor (7.5) for detection with a PMT.

the heterodyne frequency for a fixed level of local oscillator on the detector $(2 \mu W)$. Pumping the OPO with the Paladin (up to $12 \mathrm{~W}$ of $532 \mathrm{~nm}$ ) instead of our Vanguard (2.0W of $532 \mathrm{~nm}$ ) increases saturation. The decrease in the amplitude fluctuations enables shot noise limited detection for lower transimpedance (and corresponding higher frequency) until the input voltage noise dominates Johnson noise (below $50 \mathrm{k} \Omega$ ).

Figure 4 shows the measured heterodyne CARS signals for a fixed local oscillator level ( $50 n W$ for the photodiode and $2 n W$ for the PMT), where the CARS signal is varied by introducing attenuation filters in that part of the beam. For comparison we also show the direct detection of the CARS signal by removing the local oscillator and chopping the CARS beam with a mechanical chopper. The chopped signal shows the expected direct dependence on the CARS level with a slope of 1 . The heterodyne signal depends only on the root of the CARS signal and thus decreases with a slope of $1 / 2$. For increasing attenuation of the CARS level, the direct detection signal disappears in the detector noise whereas the heterodyne detection continues to lower levels before disappearing into the local oscillator noise. For detection by photodiode terminated with $10 M \Omega$, the minimum CARS signal that can be detected using the heterodyne detection is a factor 3500 lower than can be detected directly. The PMT has a very low noise level and high gain so that the improvement by the heterodyne is not so impressive (only a factor of 7.5). However, the quantum efficiency of the PMT is so low that the minimum detectable signal is considerably higher than for the heterodyne detection using a photodiode. There are PMTs with better QE commercially available (QE up to $15 \%$ are quoted). For those PMTs the heterodyne detection minimum would approach the limit for the photodiode. Due to residual alignment errors this detected heterodyne signal is a factor 10 below the theoretical limit.

The noise-free heterodyne gain is achieved for a local oscillator level that is chosen such that the local oscillator noise is only slightly above the detector dark noise level. In practice this means that the levels of local oscillator required to lift the signal above the detector noise are always fairly small $(n W)$ compared to the light levels driving the CARS $(m W)$. The local oscillator can thus be sent colinear with the other beams through a sample without disturbing 
the CARS process, avoiding path length differences in scanning microscopy.

\section{Conclusions}

For an APD the benefit from using heterodyne detection is lower since the noise levels are lower and the quantum efficiency is higher. However, it is important to note at this point that the current detection limit is not caused by detector noise alone. For very small numbers of resonant molecules in the focal volume the non-resonant background can overwhelm the resonant signal, much like auto-fluorescence limits the detection of fluorescent probes. In [7], Potma et al show that since the signal at resonance is shifted in phase compared to the non-resonant signal, the interferometric signal can be used to separate the resonant part of the CARS signal from the non-resonant part to reject this background . To avoid also noise amplification of the nonresonant signal, the polarization directions of the incoming beams and the local oscillator can be adjusted so that only the resonant part of CARS signal is amplified by the optical interference. The overall loss of signal is compensated by the interferometric gain and the noise generated by the non-resonant part does not add to the noise in the resonant part. Such a combination of Heterodyne Interference and Polarization-CARS (HIPCARS) would be analogous to the HIPSOM setup demonstrated for linear scattering by metal nanoparticles in [15].

We believe that the ability to provide noise-free amplification makes this OPO preferable to previously published sources such as continuum from nonlinear fibers $[13,14]$ where the noise levels were not specified. Also the jitter-free operation of this setup, the possibility to scan the wavelength without synchronization errors and the possibilities to distinguish resonant and non-resonant contributions will significantly enhance the applicability of CARS microscopy.

\section{Acknowledgements}

We acknowledge E.O. Potma and C.L. Evans for useful discussions during the CARS workshop 2005 at the group of X.S. Xie at Harvard. This research is supported by NanoNed, a nanotechnology programme of the Dutch Ministry of Economic Affairs and partly financed by the Stichting voor Fundamenteel Onderzoek der Materie (FOM), which is financially supported by the Nederlandse Organisatie voor Wetenschappelijk Onderzoek (NWO). We also acknowledge Coherent for the use of the Paladin laser.

\#83423 - \$15.00 USD

(C) 2007 OSA
Received 24 May 2007; revised 9 Jul 2007; accepted 28 Aug 2007; published 1 Nov 2007 12 November 2007 / Vol. 15, No. 23 / OPTICS EXPRESS 15213 